\title{
Wittgenstein's Tractatus Project as Philosophy of Information
}

\author{
R.A. YOUNG \\ Department of Philosophy, University of Dundee, Dundee DD1 4HN, UK; E-mail: \\ r.a.young@dundee.ac.uk
}

\begin{abstract}
It is argued that the Tractatus Project of Logical Atomism, in which the world is conceived of as the totality of independent atomic facts, can usefully be understood by conceiving of each fact as a bit in 'logical space'. Wittgenstein himself thinks in terms of logical space. His 'elementary propositions', which express atomic facts, are interpreted as tuples of co-ordinates which specify the location of a bit in logical space. He says that signs for elementary propositions are arrangements of names. Here, the names are understood as numerical symbols specifying coordinates. It is argued that, using this approach, the so-called 'colour-exclusion' problem, which was Wittgenstein's reason for abandoning the Tractatus, is in fact soluble. However, if logical space is a continuum then some coordinates will need to be expressed by numerical symbols that are infinite in size. How is this to be understood in Tractatus terms? It is shown that, in the Tractatus, Wittgenstein did recognise the possibility of infinite propositions and sentences expressing them. At first sight his approach to infinite sentences, and the approach of the present paper, seem to differ, but it is argued that the difference is superficial. Finally, we address the question of whether Logical Atomism is viable and this raises issues concerning its relationship to natural science.
\end{abstract}

Key words: atomism, information, limits, logic, science, Wittgenstein

\section{Information and Elementary Propositions}

This paper explores Wittgenstein's project of Logical Atomism in the Tractatus and shows how it can be understood as Philosophy of Information. It explores the idea that an elementary proposition in the Tractatus is to be understood as one bit of information. Intuitively a bit is just the smallest unit of information. In truth tables, which Wittgenstein uses ${ }^{1}(1922,4.31)$, we could think of a truth value assignment to a proposition with a $\mathrm{T} / \mathrm{F}$ as the equivalent to a bit. However, we shall also think of a bit in binary arithmetic as 1 or a 0 . In the physical world, we can think of it as equivalent to a switching state being turned on or off.

Consider the following propositions from close to the beginning of the Tractatus (Wittgenstein, 1922):

1.1 The world is the totality of facts, not of things.

1.11 The world is determined by the facts, and by these being all the facts.

1.12 For the totality of facts determines both what is the case, and also all that is not the case.

1.13 The facts in logical space are the world.

1.2 The world divides into facts. 
1.21 Any one can either be the case or not be the case, and everything else remain the same.

Here Wittgenstein envisages 'atomic' facts, and they are each to be independent of each other as envisaged in 1.21. These atomic facts seem to be bits.

However, Wittgenstein does say that signs for elementary propositions consist in arrangements of names and correspondingly that atomic facts are arrangements of simple objects. Thus a propositional sign seems to consist of more than one bit. How does this fit in? Perhaps we can develop an answer by considering logical space, which is mentioned in 1.13 and elsewhere in the Tractatus $(1.13,2.11,2.202$, $2.21,3.4,2.42,4.463)$. Think of a position in logical space as being occupied if a bit is switched on. An elementary proposition needs to show us which bit is to be switched on to make it true. It can do this by giving us the coordinates of that bit in logical space. In that case, the numerical signs for coordinates in logical space name simple objects and those simple objects are coordinates in logical space. Consider:

3.4 The proposition determines a place in logical space: the existence of this logical place is guaranteed by the existence of the constituent parts alone, by the existence of the significant proposition.

3.41 The propositional sign and the logical co-ordinates: that is the logical place.

On the interpretation I am following, the constituent parts of an elementary proposition are its logical coordinates. The difference between the propositional sign in an elementary proposition and the signs for the coordinates is just that the elementary proposition consists of a tuple (sequence of a certain length - an arrangement) of signs for coordinates. We need to consider whether this interpretation of objects makes sense in the Tractatus, consider:

2.021 Objects form the substance of the world. Therefore they cannot be compound.

2.0211 If the world had no substance, then whether a proposition had sense would depend on whether another proposition was true.

2.0212 It would then be impossible to form a picture of the world (true or false).

2.022 It is clear that however different from the real one an imagined world may be, it must have something - a form - in common with the real world.

2.023 This fixed form consists of the objects.

Is it odd to treat coordinates as simple objects? Are not coordinates somehow compound? What is necessary in Tractatus terms to confer simplicity on objects is that the facts constituted out of them satisfy the requirement imposed in 2.2011, which requires that atomic facts be independent of each other as required in 1.21. The thesis of the present paper is that bits in logical space do have this independence. In defence of the objects-as-coordinates interpretations, it may be argued that it can give us an intuitive 'fixed form' for the world as envisaged in 2.023. Of course, one 
could think in terms of a possible-worlds semantics in which the same domain is used throughout, and in terms of facts about individuals being absent from worlds. However, it is questionable whether the mere existence of a common domain constitutes a 'fixed form' for the world, thus the objects-as-coordinates interpretation presented here is preferable.

\section{Some Initial Objections}

The syntax that is proposed in the present paper for signs for elementary propositions is simply that they are arrangements of numerical symbols for coordinates. If we take symbols for coordinates to be names of 'objects' then the interpretation fits well with what Wittgenstein says about propositions being arrangements of names of objects (op. cit. 3.21). Indeed the proposal unifies these aspects of what he says with his remarks about logical coordinates. Consider what an elementary proposition for a two-dimensional geometrical space and one extra 'property' dimension would look like in a syntax using bracketing and commas. Consider for example, a propositional sign meaning at the point, which is 2 on the $x$-axis and 2 on the $\mathrm{y}$-axis, the second property bit is on:

\section{$(2,2,2)$}

In this syntax, as well as the 'names' and their spatial arrangement, we have marks to demarcate one argument from another (commas) and also one proposition from another (brackets). Clearly we need some conventional way of demarcating arguments and propositions, but there might be many conventions.

At first sight, objections to our proposal can be generated from the Tractatus itself. One objection concerns the syntactic constraints explicitly proposed in the Tractatus. Another objection concerns the semantics. A third concerns topology. A fourth objection is that we are naming numbers, but Wittgenstein employs other expressions for numbers $(1922,6.021)$. The syntactic objection is very simple. Wittgenstein declares that there is to be a one-to-one pairing of names and objects, thus eliminating the need for an identity sign: identity of object is to expressed simply by using the same sign (op. cit. 5.53). Yet, in the syntax we propose, what appears to be the same name, for example ' 2 ' in our example, can refer to different objects. However, this objection does not have much force. The way to read our coordinate syntax is that position is part of what constitutes a name, thus in our example, although ' 2 ' appears thrice, it constitutes a different syntactic name on each occasion, because a name is a combination of digit(s) and position. Of course, to indicate the use, we would introduce an alphabetical component of our syntactic name, and use the numerical symbols as a subscript, thus ' 2 ' in the first argument place might become ' $a_{2}$ '. The semantic objection is that coordinates are not 'objects', which they should be according to the Tractatus. This is a very weak objection. The Tractatus requires peculiar objects, because the objects are 
to constitute the 'form' of the world, which is the same in every possible world. Coordinates, which I take to be positions with respect to an axis, seem eminently suited to the fulfilment of what the Tractatus requires. The topological objection to our proposal for 'names' is that, since space may curve back on itself, many different symbols for coordinates (for example on the $x$-axis) may refer to exactly the same position on the $x$-axis, each coordinate reflecting another circuit around the space. However, this depends upon the conventions we adopt. We may refuse to apply a repetitive naming convention to a curved space. Another conventional way out of the topological problem is to insist on treating all curved spaces as being spaces embedded in a large Euclidean space. The objection that numbers are being named is also weak; coordinates are being named not numbers and we could move the numerical symbols to a subscript to indicate this as we have illustrated with $a_{2}$.

As well as the objections to which I have just responded, which are objections to my interpretation rather than to elementary propositions themselves, there are what I would regard as conceptual objections either (i) to the very idea of elementary propositions or (ii) to the specific idea of them proposed in this paper. An example of (i) is the well-known objection to elementary propositions that is known as the colour-exclusion problem.

\section{The Colour-Exclusion Problem}

By definition, in the Tractatus, the truth or falsity of each elementary proposition is to be entirely independent of that of all others. We are intended to be able to know a priori that there are such elementary propositions and that all other propositions are analysable in terms of them. Indeed we are to know these truths because they are tautological. Thus it is to arise from the very nature of symbolism itself that such elementary propositions will inevitably be articulable for any language and that all other propositions will be analysable into them. Is it conceivable that these very strong claims can be justified? The standard view is that they cannot be. This is because of the problem that is known as the colour-exclusion problem. Colour properties are discussed in the Tractatus $(2.0251,2.031,2.171,4.123,6.3751)$ itself. Ramsey in the first review of it (1923) raises the problem. It is often understood as the problem that something cannot simultaneously have two pure colours (e.g. something that is purely red cannot be purely blue). However, this is not a good statement of the problem. Suppose we introduce a sense of red in which something is 'red' so long as there is some red in it (so orange is red for example), and we introduce corresponding senses of green and blue. Now, once we analyse 'purely', the problem that something cannot be purely red and purely blue disappears. This is because, ' $a$ is purely red' can be expressed by ' $\neg B a \& \neg G a \& R a$ ' whereas ' $a$ is purely blue' can be expressed as ' $B a \& \neg G a \& \neg R a$ '. On this analysis, the problem disappears because the basic colour predicates are logically independent of each other and inconsistency only arises if we attempt to assert a colour predicate and simultaneously negate it. However, the problem with this is that if we cannot 
specify degrees of red, green and blue, then we cannot specify the various shades of mixed colour precisely. Once we introduce degrees of red, blue and green, the problem recurs. If there are six degrees of red then this is inconsistent with there being five degrees of red. However, once we have recast the problem in this form, then we can see that the problem is one of dealing with quantity or degree.

Wittgenstein himself used colour-exclusion to argue against his Tractatus view (1966, pp. 35-36 ). If Wittgenstein were entirely correct in his later criticisms, then no solution of his Tractatus problem would be possible. In the present section, I shall show that his later criticisms of the Tractatus - at least those based on the colour-exclusion problem - were mistaken. Perhaps what misled him was that he knew that when he wrote the Tractatus he did have in mind a mistaken 'solution' to the colour-exclusion problem. However, if he did have a 'solution', there is some controversy as to what it was. Ramsey (1923) thought that his solution was to be by reducing colour to the motion of physical particles and this is endorsed by Monk (1991, p. 273). They base this view on Tractatus 6.3751, but, so far as I can see, in that proposition Wittgenstein is simply drawing attention to the fact that there are problems concerning the motion of physical particles that parallel the colour-exclusion problem. In any case, Ramsey and Monk only propose their interpretation in order to accuse Wittgenstein of confusing logic with physics. James Austin (1980) has another interpretation of the Tractatus solution, which also consists in attributing a logically very defective analysis to Wittgenstein (albeit it may be no worse than the 'solution' Ramsey and Monk attribute). The solution is supposed to be that colour is analysed into degrees of red, green and blue (this is, of course, perfectly standard). Then degrees of red are treated as multiples of unit redness. Finally, there is an attempt to analyse unit redness in terms of the logical product of atomic propositions each of which attribute one unit of redness. Thus, on Austin's account, if $R(a)$ meant that a had one unit of redness then ' $R(a)$ and nothing else' would express that $a$ has one unit of redness, ' $R(a)$ and $R(a)$ and nothing else' would express that $a$ had two units of redness and so on. Austin points out this is very defective, because the repetition of ' $R(a)$ ' just asserts the very same thing over again. Also there is the problem of the general proposition expressed by 'nothing else'. How exactly would that be analysed?

Perhaps the most plausible interpretation of Wittgenstein, is that when he wrote the Tractatus he had the account of logical space that we have proposed, but he did not have a solution to the colour-exclusion problem, just a conviction based on general arguments that it was soluble. In 1929 and later, Wittgenstein had been convinced by Ramsey that the colour-exclusion problem was insoluble; this is why he abandoned the Tractatus position and tried to develop alternatives. Be that as it may, there is a solution to the problem along Tractatus lines, which is not logically defective, and which is closely related to modern digital analyses of colour. We can solve the problem by using the coding for colour that is used in modern computer systems, and then re-expressing it in what, following Wittgenstein, we may call elementary propositions. For simplicity, consider how 8-bit colour is represented on the BGR233 system. We have two bits available for blue, three for green, and 
three for red. This gives us four degrees of colour for blue (00,01,10 and 11), eight degrees for green $(000,001,010,011,100,101,110$ and 111) and similarly eight for red, giving 256 colours in all. A given colour might be represented as 01111011. Each of the bits in the 8-bit sequence is logically independent of the other bits. Thus we can analyse colour into logically independent atomic facts each of which is just the fact that a particular bit is turned on in the complex colour fact. Suppose I am to represent the colour at a point on a two dimensional plane. I can do this by using three coordinates, an $x$ coordinate to represent position along the $x$-axis, a y coordinate to represent position along the $y$-axis and a third coordinate which will tell me which bit I am making an assertion about. Thus, if I use 1 as the name for the first colour bit, 2 as the name for the second colour bit and so on . . . , then I can assert that the first colour bit is turned on at spatial point $(2,3)$ with the arrangement of coordinates: $(2,3,1)$. If I want to assert that the bit is not turned on, and I use $\neg$ to represent negation, then I say $\neg(2,3,1)$. We can compare different representations of the fact that spatial point $(2,3)$ has a certain predominantly green colour as follows:

English: The point which is 2 along the $\mathrm{x}$-axis and 3 up the y-axis is yellowygreen.

Binary representation of 8-bit colour: The point $(2,3)$ has colour 01111011

Representation by truth function of logical atoms: $\neg(2,3,1) \&(2,3,2) \&(2,3$, $3) \&(2,3,4) \&(2,3,5) \& \neg(2,3,6) \&(2,3,7) \&(2,3,8)$

This representation does give us elementary propositions that are logically independent of each other, as the Tractatus requires. It represents colour in space essentially by adding an extra dimension to geometrical space, but one which represents a bitwise decomposition of colour. It can be argued that the representation is very close to the representation proposed in the Tractatus for atomic facts, because Wittgenstein does talk of a colour space (2.031, see also 2.0251).

Moreover, it should be clear that what has been done here for colour could be done for any other property identifiable by a coordinate, where the coordinate is one of a range all of which identify degrees of the property, because the technique is simply to express in binary the number used to quantify the coordinate and then to generate atomic propositions about whether each bit is turned on or not. With this way of representing properties, it is possible to argue that its applicability follows from the nature of symbolism itself on the ground that anything expressible in a language can be given a model in a space describable in the proposed language of Logical Atomism. Indeed, if this solution succeeds, then it does so by analysing the information provided in propositions into basic units of information.

However, it would seem that the coding that we have proposed, even though it meets Tractatus requirements, cannot be regarded as Wittgenstein's Tractatus coding, because if it had been, he would not have been perturbed by Ramsey's colour-exclusion criticism. At least he would not, unless, during his period away from philosophy, he had simply forgotten the Tractatus solution. We can think of 
the proposed coding as carrying through the project of the Tractatus. It provides us with a rational reconstruction of what Wittgenstein would have thought about the colour-exclusion problem, if he had continued with the Tractatus project, and had become aware of the bitwise analysis of colour.

In any event, we need to recognise that the proposed solution, or rational reconstruction, can be challenged, albeit not by Ramsey's argument. Suppose that we use the 8-bit colour coding given above, but then we find that we need 257 colours rather than 256, because there are 257 discriminable colours. In the 'atomic' coding that we have discussed, this will necessitate moving to 512 colours instead of 256 . We cannot block this, because in the 'atomic' coding each bit is given an independent meaning and so if we go up to 9 bits we have 512 compound propositions to form. Should we regard this as a cogent objection to our proposed solution to the colour-exclusion problem? To answer this question, it is helpful to consider the approaches to colour that Wittgenstein himself considered after he had abandoned the Tractatus position.

When Wittgenstein abandoned the Tractatus position, he adopted first of all an intermediate 'solution', and then his eventual 'solution'. In both of these, he claimed that the whole colour system could be placed against reality 'like a ruler', and that it would be mandatory in the system that colours would be incompatible. In the case that there were 257 discriminable colours, the ruler could, as it were, have 257 marks on it. In the intermediate solution (Wittgenstein 1966), the size of the ruler was to be determined by phenomenological investigation. If Wittgenstein had been content with that, and the phenomenology was identified by a philosophical method, then he would have been forced to abandon his critique of philosophy, because philosophy would have had a special phenomenological means of investigation. Alternatively, if the phenomenology were identified by empirical psychological investigation, then he would have been forced to abandon his critique of empirical psychology. Instead of this intermediate position, the later Wittgenstein proposed (1975, pp. 105-114 and p. 317) that the number of colour terms was regulated by what he called 'grammar', i.e. the rules of language. In this event, it seems that if we had a grammar which sanctioned 256 colour terms, then we would be justified in maintaining it even though we were capable of recognising 257 colour distinctions. It is not obvious, once it is recognised that the colourexclusion problem can be overcome, that Wittgenstein's eventual position is an improvement on the Tractatus-like position that we have developed. If we are to let 'grammar' determine the number of colours, why not let 'grammar' sanction 512 terms even though we will ever only apply 257 ?

\section{Infinite Propositions}

Is it a reasonable objection to elementary propositions, as envisaged in this paper, that they might not be finitely expressible, because coordinates may only be expressible using numerical symbols for non-computable real numbers, to name 
them all would require names of infinite length, and so at least some propositional signs would need to be infinite? It is clear that Wittgenstein in the Tractatus did not object to propositional signs of infinite length. Thus he says (1922, op. cit.):

4.2211 Even if the world is infinitely complex, so that every fact consists of an infinite number of atomic facts and every atomic fact is composed of an infinite number of objects, even then there must be objects and atomic facts.

This makes the Tractatus language, at least potentially, a God's eye language, not one we could write or speak, yet one for which we are supposed to see the need. ${ }^{2}$

It must be admitted that Wittgenstein's ostensible reason for admitting the possibility of infinite propositions is not the reason that arises in the interpretation that this paper has developed of the Tractatus. Wittgenstein countenances infinite relations, whereas the interpretation provided by the present paper countenances infinite names. This difference will be resolved at the end of the present section, but first let us understand Wittgenstein's proposal in its own terms.

We need to clarify in what sense the world might consist of infinitely complex atomic facts. Wittgenstein says that whether we need to have relations of a given arity is not a matter for logic, but for its application.

5.5541 How could we decide a priori whether, for example, I can get into a situation in which I need to symbolize with a sign of a 27 -termed relation?

5.557 The application of logic decides what elementary propositions there are. What lies in its application logic cannot anticipate.

It is clear that logic may not con ict with its application.

But logic must have contact with its application.

Therefore logic and its application may not overlap one another.

Thus in logic, we can understand that there is no formal limit on the number of terms that a relation might have. However, in a language that we apply to the world, the maximum size of relation ${ }^{3}$ that we might need to express in an atomic proposition depends upon the applications that we have discovered and these will be limited by the world.

If we could know how they were limited, then we could know the maximum size of relation, but at any given stage in our enquiries we will only so far have given applications to relations of a certain size, and it is Wittgenstein's position that we cannot know whether we might need to give applications to relations of a larger size. Indeed there is no guarantee that we could ever come to an end with a largest size of relation. Thus it seems that logic cannot settle the issue of whether, at the limit, there might be a need for an infinite relation, and nor can any human finite application of language.

It may seem that the idea that we cannot know the maximum arity of relation is in conflict with Wittgenstein's notion that the whole of logical space is implicit in any one proposition (Wittgenstein, 1922, 3.42). However, the idea is that, on a full 
analysis of any everyday proposition, the whole of logical space is implicit, but it does not follow that we know what all the implications are, because we may well not yet have explicitly applied language in a way that enables us to articulate a fully explicit analysis. In everyday language, we do not explicitly employ Wittgenstein's elementary propositions. Indeed Wittgenstein says (op. cit.):

4.002 . . . Language disguises the thought; so that from the external form of the clothes one cannot infer the form of the thought they clothe, because the external form of the clothes is constructed with quite another object than to let the form of the body be recognized. ...

If we could apply language at the limit, then each of its propositional signs would express fully determinate propositions. Indeed in Wittgenstein's view each proposition of everyday language is 'logically completely in order' (Wittgenstein 1922, 5.5563). That is to say, everything which counts as a proposition in our everyday language is logically completely in order, but, of course, this is perfectly consistent with very many would-be propositional signs in everyday language being nonsense. According to Wittgenstein, what we can know is that there are elementary propositions which express atomic facts (Wittgenstein, 1922, 5.5562), because this follows by logical reasoning from any language that has an application, but it does not follow from this that we can know what elementary propositions there are.

Given this account of elementary propositions, we can now proceed to consider Wittgenstein's notion that there might be many-term relations (e.g., the 27-term relation that he considers) and then after that we will consider his notion that there might be elementary propositions of infinite complexity, in the sense of consisting of an infinity of names and thus involving an infinite relation.

First, consider the idea that elementary propositions might involve many-term relations, in the sense of relations that are of greater arity than binary relations. We need to consider, if only to dismiss it, a simple argument against there being fundamental relations of arity greater than two. This is that any proposition that a relation of greater arity obtains can be converted into a compound proposition asserting that corresponding binary relations hold always provided that we introduce an extra term that, as it were, links these binary relations together. This conversion is well known in philosophy from a paper by Davidson (2001, pp. 105-122), in which he argues that even though actions might appear to involve many-term relations they should be universally analysed using an extra term for an event and then binary relations of being the agent of that event, being (in the case of a transitive verb) the patient of the event, being the place of the event and so on.

From the Tractatus perspective, this transposition from many-placed predicates into binary does not guarantee that the propositions using binary relations will be atomic. Thus, if we have a true elementary proposition that says something is the patient of an event then there must also be a true proposition which says that that event has an agent. In particular, if we consider signs for elementary propositions to be arrangements of signs for coordinates, and try to transpose to binary relations 
as proposed, then we might say that a certain named event has a certain position on the $x$-axis, say 6 , which would be inconsistent with saying that it has a different position, say 7. Now that we have dismissed the proposed transposition to binary relations as unacceptable in Tractatus terms, we can turn to the question of infinity.

We can begin by considering elementary propositions with a denumerable infinity of terms. With coordinates as names, that would mean that logical space would have an infinity of dimensions. In the interpretation proposed in this paper, dimensions are of two distinct kinds, there are property dimensions, such as the dimension of colour in our example, and then there are the dimensions of geometrical space-time. As can be seen from our example of colour, an elementary proposition need only involve one property dimension for which we say that a certain bit is set. Admittedly, since we need to distinguish between different property dimensions, we will need a coordinate to identify the property dimension and another coordinate to identify which bit in that dimension, we are asserting to be set. To return to our example, if there are two dimensions of geometrical space, the colour dimension is the first property dimension and we use 1 to represent it, then, if we wish to say that the second bit of the colour dimension is set for the geometrical position $(3,4)$, we can do that as follows:

$$
(3,4,1,2)
$$

There might be an infinity of other property dimensions and yet we would not need to mention them. Thus it seems at first sight that, if there is to be a need for elementary propositions with an infinity of names, then this will be because we have an infinite number of geometrical dimensions.

However, there is a different way of understanding how we might need propositions each of which uses an infinity of names. This way reconciles the interpretation proposed in the present paper with what Wittgenstein says about an infinity of names. Consider that in each of the geometrical dimensions, or even in a property dimension, we might have a continuum. In that case, the coordinate in the continuum might need to be expressed using numerical signs for a real number, that is a non-computable ${ }^{4}$ real number. In this event, there is an alternative way of identifying propositions, in which we do not name precise coordinates in the continuum but instead name interval-coordinates, but thus there is an infinity of coordinates. Suppose we are have just two geometrical dimensions, but each is a continuum. In our proposed symbolism, each of these coordinates will be expressed using the symbol for a real number. The alternative symbolism is to use an infinite number of finitely expressible 'interval' coordinates as follows. First consider, the integer component of each real number, each of these can be expressed using a finite numerical symbol and these can be the first two interval coordinates in our alternative symbolism. Now consider the fractional component of each real number. We can restrict each name for an interval 'coordinate' to some finite number of digits, say 1, but use an infinity of interval coordinates as follows: the first pair of interval coordinates corresponds to the first digit in each of the fractional signs for real numbers for the two dimensions, the next pair is used for the second digits 
and so on. Thus the infinite sequence of every other digit after the first expresses the $x$-axis and the infinite sequence of every other digit after the second expresses the $y$-axis. Each digit in the first sequence represents an interval on the $x$-axis, and each digit in the second sequence represents an interval on the $y$-axis. A motivation for this coding is that each interval coordinate can be finitely symbolised by us but we could not finitely name each precise coordinates in the continuum.

\section{Can the Tractatus Project Be Carried Forward?}

The Tractatus, despite its brevity, is extremely complex. There are some parts of it that cannot be sustained, because of what we have learned since Tractatus times, for example about logic and computation. Thus we are told:

6.126 Whether a proposition belongs to logic can be calculated by calculating the logical properties of the symbol. And this we do when we prove a logical proposition. ...

In the 1930s it was discovered that predicate logic is not decidable, and thus this claim of the Tractatus is wrong.

The present paper does not aim to defend every claim made in the Tractatus. The aim of this paper is to consider whether the Tractatus Project of Logical Atomism can be understood, or rationally reconstructed as, philosophy of information, and, if so, whether it is defensible. We have seen that a bitwise analysis of colour can solve the colour-exclusion problem, and that a bitwise approach can be coherent with the Tractatus in its approach to infinity.

However, a further question is whether Logical Atomism can be sustained against all objections. We have come close to a certain kind of conventionalism. In effect, it has been proposed that we can adopt a convention of interpreting any way of representing the world in a form of bitwise coding. For example, if we code colour as proposed in section 3, then the number of different shades of colour will double for each bit in the colour dimension, so, if we could only discriminate an odd number of colours (257 in our earlier example), nevertheless we would be forced to identify, through our symbolism, a number of shades that equated to the next power of 2 (512 in our example). Thus, arguments might be presented for and against bitwise coding, property by property, for scientific or other reasons.

In the Tractatus, an analogy is drawn between logic and science (or mechanics to be precise) and a certain conventionalism is adopted for science. Pure Logical Atomism, in an infinitary language, would specify all the points that make up the spots in 6.341 below, but mechanics conventionally requires one form of representation or another:

$6.341 \ldots$ Let us imagine a white surface with irregular black spots. We now say: Whatever kind of picture these make I can always get as near as I like to its description, if I cover the surface with a sufficiently fine square network and now say of every square that it is white or black. ... This form 
is arbitrary, because I could have applied with equal success a net with a triangular or hexagonal mesh. . . . To the different networks correspond different systems of describing the world. Mechanics determine a form of description by saying: All propositions in the description of the world must be obtained in a given way from a number of given propositions - the mechanical axioms. ...

6.342 And now we see the relative position of logic and mechanics. . . That a picture ... can be described by a network of a given form asserts nothing about the picture.... But ... [what] characterize[s] the picture ... [is] that it can be completely described by a definite net of definite fineness.

So too the fact that it can be described by Newtonian mechanics asserts nothing about the world; but this asserts something, namely, that it can be described in that particular way in which as a matter of fact it is described. The fact, too, that it can be described more simply by one system of mechanics than by another says something about the world.

On this account, the forms we adopt in science maybe conventional, but perhaps there may nevertheless be reasons for preferring one form or formal theory to another. Whether this conventionalism can be sustained is a question that touches on complicated matters. Wittgenstein's Tractatus was written in the days of the old Quantum Theory. The new Quantum Theory, with its challenge to Newtonian Mechanics had not yet arrived. ${ }^{5}$

However, in order to defend the solution to the colour-exclusion problem that we have proposed, we have embraced a certain conventionalism. So might our proposed position be discarded for scientific reasons? In any case, it may be argued that Quantum Theory challenges Logical Atomism, or at least the Tractatus account of it. There seem to be two challenges to the Tractatus in the new theory. The first is that some propositions describing observables seem to be neither true nor false, which con icts with such propositions as:

6.111 Theories which make a proposition of logic appear substantial are always false. One could, e.g., believe that the words "true" and "false" signify two properties among other properties, and then it would appear as a remarkable fact that every proposition possesses one of these properties.

. . . Indeed our proposition now gets quite the character of a proposition of natural science and this is a certain symptom of its being falsely understood.

The second is that it is questionable whether descriptions of non-observable quantum states have sense independently of each other. In classical physics, there might in principle be measurements of all the properties described in the theory and thus in principle the application of the symbolism to describe one point in space- 
time might be independent of its application to describe another, but this is not so in quantum mechanics. On the basis of the new Quantum Theory, classical logic has even been challenged, however it seems we are not forced to abandon classical logic because of it (van Fraassen, 1991, pp. 134-135), but that does not in itself guarantee that an acceptable Tractatarian interpretation of Quantum Mechanics can be given. Currently computational approaches are being developed towards Quantum Mechanics itself (Deutsch, 2002). The Tractatus approach, even reconstructed as in the present paper, is bitwise rather than computational (in principle, bitwise changes could implement non-computable functions). ${ }^{6}$ Thus, the feasibility of a computational approach to Quantum Mechanics does not guarantee the independence of one fact from another that we have in Logical Atomism. The crucial issue here is whether each elementary propositional sign can be given an independent sense in a way that enables every combination of truth value assignments to elementary propositions to have a clear meaning.

\section{Conclusion}

It has been argued that the Tractatus Project of Logical Atomism, can usefully be understood by conceiving of each fact as a bit in 'logical space'. Wittgenstein's 'elementary propositions', which express atomic facts, have been interpreted as tuples of coordinates which specify the location of a bit in logical space. Here, the names are understood as numerical symbols specifying coordinates. It has been argued that, using this approach, the so-called 'colour-exclusion' problem, which was Wittgenstein's reason for abandoning the Tractatus, is in fact soluble. However a further problem arises, if logical space is a continuum, and thus symbols for coordinates need to be infinite in size. We have seen that, in the Tractatus, Wittgenstein did recognise the possibility of infinite propositions and sentences expressing them. It has been shown that we can re-express propositions which employ infinite names as propositions which employ an infinity of names. Thus the interpretation, or rational reconstruction, proposed in this paper does turn out to be coherent with Wittgenstein's own position on infinite sentences. The question of whether Wittgenstein's Logical Atomism could be sustained in contemporary debate has been raised, but a full answer to this question is beyond the scope of the present paper.

\section{Notes}

\footnotetext{
${ }^{1}$ Indeed he helped to introduce them.

${ }^{2}$ Perhaps this aspect of the language is even connected with his remarks about ethics at the end of the Tractatus, but the present paper is not concerned to develop that aspect of his thought.

${ }^{3}$ It might seem to some logically-minded readers that it is possible to analyse any propositions with relations of arity greater than two into propositions that have to do entirely with binary relations. This is dealt with later.
} 
${ }^{4}$ It may be urged that it is anachronistic with respect to the Tractatus to introduce considerations of computability. There is some force in this objection, but it should not rule computability entirely out of question - the continuum was understood before the Tractatus was written, and the concept of an algorithm was a longstanding concept.

${ }^{5}$ See Kragh (1999) for an account of the relevant history of science.

${ }^{6}$ Quantum computation affords special kinds of efficiency, but a quantum computer would not compute any any functions beyond those computable on a universal Turing machine.

\section{References}

Austin, J. (1980), 'Wittgenstein's Solutions to the Colour Exclusion Problem', Philosophy and Phenomenological Research 41 (1-2).

Davidson, D. (2001), Essays on Actions and Events, 2nd edn, Oxford University Press.

Deutsch, D. (2002), 'The Structure of the Multiverse', Proc. R. Soc. Lond. 458 (2028), pp. 2911-2923.

Kragh, H. (1999), Quantum Generations, Princeton: Princeton University Press.

Monk, R. (1991), Ludwig Wittgenstein, the Duty of Genius, Vintage.

Ramsey, F. (1923), 'Tractatus Logico-Philosophicus', Mind 32 (128), pp. 465-478.

van Fraassen, B. (1991), Quantum Mechanics, O.U.P.: Oxford.

Wittgenstein, L. (1922), Tractatus Logic-Philosophicus, New York: Keagan Paul.

Wittgenstein, L. (1966), 'Some Remarks on Logical Form', in Copi and Beard, eds, Essays on Wittgenstein's Tractatus, New York: Routledge and Kegan Paul, pp. 31-37.

Wittgenstein, L. (1975), Philosophical Remarks, Blackwell, trans. Hargreaves and White. 